\title{
On Quasi Lindley Distribution and Its Applications to Model Lifetime Data
}

\author{
Rama Shanker $^{1}$, Hagos Fesshaye ${ }^{2}$, Shambhu Sharma ${ }^{3}$ \\ ${ }^{1}$ Department of Statistics, Eritrea Institute of Technology, Asmara, Eritrea \\ ${ }^{2}$ Department of Economics, College of Business and Economics, Halhale, Eritrea \\ ${ }^{3}$ Department of Mathematics, Dayalbagh Educational Institute, Dayalbagh, Agra, India
}

\section{Email address:}

shankerrama2009@gmail.com (R. Shanker),hagosfisha@gmail.com (H. Fesshaye), ssdei61@gmail.com (S. Sharma)

\section{To cite this article:}

Rama Shanker, Hagos Fesshaye, Shambhu Sharma. On Quasi Lindley Distribution and Its Applications to Model Lifetime Data. International Journal of Statistical Distributions and Applications. Vol. 2, No. 1, 2016, pp. 1-7. doi: 10.11648/j.ijsd.20160201.11

Received: March 3, 2016; Accepted: March 22, 2016; Published: April 14, 2016

\begin{abstract}
In this paper mathematical and statistical properties including moment generating function, mean deviations about mean and median, order statistics, Bonferroni and Lorenz curves, Renyi entropy and stress strength reliability of quasi Lindley distribution (QLD) introduced by Shanker and Mishra (2013 a) have been derived and discussed. The goodness of fit of QLD over exponential and Lindley distributions have been illustrated with five real lifetime data-sets and found that QLD provides better fit than exponential and Lindley distributions.
\end{abstract}

Keywords: Mean Deviations, Order Statistics, Bonferroni and Lorenz Curves, Renyi Entropy Measure, Stress-Strength Reliability, Goodness of Fit

\section{Introduction}

Lindley distribution, introduced in the context of Bayesian analysis as a counter example of fiducial statistics, having probability density function (p.d.f) and cumulative distribution function (c.d.f)

$$
\begin{aligned}
& f(x ; \theta)=\frac{\theta^{2}}{\theta+1}(1+x) e^{-\theta x} \quad ; x>0, \theta>0 \\
& F(x ; \theta)=1-\left[1+\frac{\theta x}{\theta+1}\right] e^{-\theta x} ; x>0, \theta>0
\end{aligned}
$$

has been introduced by Lindley (1958). A detailed study about its important mathematical and statistical properties, estimation of parameter and application showing the superiority of Lindley distribution over exponential distribution for the waiting times before service of the bank customers has been done by Ghitany et al (2008). The Lindley distribution has been generalized, extended, modified and mixed with other discrete distributions by different researchers including Zakerzadeh and Dolati (2009), Nadarajah et al (2011), Deniz and Ojeda (2011), Bakouch et al (2012), Shanker and Mishra (2013 a, 2013 b), Shanker and Amanuel (2013), Shanker et al (2013), Elbatal et al (2013), Ghitany et al (2013), Merovci (2013), Liyanage and Pararai (2014), Ashour and Eltehiwy (2014), Oluyede and Yang (2014), Singh et al (2014), Sharma et al (2015), Shanker et al (2015 a, 2015 b), Alkarni (2015), Pararai et al (2015) are some among others.

The probability density function (p.d.f.) and cumulative distribution function (c.d.f) of quasi Lindley distribution (QLD) of Shanker and Mishra (2013a) are given by

$$
\begin{aligned}
& f(x ; \alpha, \theta)=\frac{\theta}{\alpha+1}(\alpha+\theta x) e^{-\theta x} ; x>0, \theta>0, \alpha>-1 \\
& F(x ; \alpha, \theta)=1-\left[\frac{1+\alpha+\theta x}{\alpha+1}\right] e^{-\theta x} ; x>0, \theta>0, \alpha>-1
\end{aligned}
$$

At $\alpha=\theta$, both (1.3) and (1.4) reduce to the corresponding expressions (1.1) and (1.2) of Lindley distribution. The first two moments about origin and the variance of QLD obtained by Shanker and Mishra (2013a) are

$$
\mu_{1}^{\prime}=\frac{1}{\theta}\left(\frac{\alpha+2}{\alpha+1}\right)
$$




$$
\begin{aligned}
& \mu_{2}^{\prime}=\frac{2}{\theta^{2}}\left(\frac{\alpha+3}{\alpha+1}\right) \\
& \mu_{2}=\frac{\alpha^{2}+4 \alpha+2}{\theta^{2}(\alpha+1)^{2}}
\end{aligned}
$$

At $\alpha=\theta$, these moments reduce to the corresponding moments of Lindley distribution. Shanker and Mishra (2013 a) have derived and discussed some of its mathematical properties including its shape, moments, coefficient of variation, coefficient of skewness and kurtosis, hazard rate function, mean residual life function and stochastic orderings. They have also discussed the estimation of its parameters using maximum likelihood estimation and method of moments and its goodness of fit over Lindley and exponential distributions. It has been observed that many important mathematical and statistical properties of this distribution have not been derived and studied.

In the present paper some of the important mathematical and statistical properties including moment generating function, mean deviations about mean and median, order statistics, Bonferroni and Lorenz curves, Renyi entropy measure and stress strength reliability of QLD of Shanker and Mishra (2013 a) have been derived and discussed. Its goodness of fit over exponential and Lindley distributions have been illustrated with some real lifetime data-sets and found that QLD gives better fit than exponential and Lindley distributions.

\section{Mathematical and Statistical Properties}

\subsection{Moment Generating Function}

The moment generating function, $\left(\mathrm{M}_{\mathrm{X}}(\mathrm{t})\right)$ of QLD (1.3) can be obtained as

$$
\begin{gathered}
M_{X}(t)=\frac{\theta}{\alpha+1} \int_{0}^{\infty} e^{-(\theta-t)}(\alpha+\theta x) d x=\frac{\theta}{\alpha+1}\left[\frac{\alpha}{\theta-t}+\frac{\theta}{(\theta-t)^{2}}\right] \\
=\frac{\theta}{\alpha+1}\left[\frac{\alpha}{\theta} \sum_{k=0}^{\infty}\left(\frac{t}{\theta}\right)^{k}+\frac{1}{\theta} \sum_{k=0}^{\infty}\left(\begin{array}{c}
k+1 \\
k
\end{array}\right)\left(\frac{t}{\theta}\right)^{k}\right] \\
=\sum_{k=0}^{\infty}\left(\frac{\alpha+k+1}{\alpha+1}\right)\left(\frac{t}{\theta}\right)^{k}
\end{gathered}
$$

It can be easily seen that the expression for $\mu_{r}^{\prime}$, obtained as the coefficient of $\frac{t^{r}}{r !}$ in $M_{X}(t)$ is given as

$$
\mu_{r}^{\prime}=\frac{r !(\alpha+r+1)}{\theta^{r}(\alpha+1)} ; r=1,2,3, \ldots
$$

For $\alpha=\theta, \mu_{r}^{\prime}$ reduces to the corresponding $\mu_{r}^{\prime}$ of Lindley distribution. For $r=1$ and $r=2$ the first two moments about

origin as given by (1.5) and (1.6) can easily be obtained.

\subsection{Mean Deviations about Mean and Median}

The amount of scatter in a population is measured to some extent by the totality of deviations usually from their mean and median and are known as the mean deviation about the mean and the mean deviation about the median, and are defined as

$$
\delta_{1}(X)=\int_{0}^{\infty}|x-\mu| f(x) d x \text { and } \delta_{2}(X)=\int_{0}^{\infty}|x-M| f(x) d x
$$

respectively, where $\mu=E(X)$ and $M=\operatorname{Median}(X)$. The derivation of measures $\delta_{1}(\mathrm{X})$ and $\delta_{2}(\mathrm{X})$ can be obtained using the following simplified relationships

$$
\begin{aligned}
& \delta_{1}(X)=\int_{0}^{\mu}(\mu-x) f(x) d x+\int_{\mu}^{\infty}(x-\mu) f(x) d x \\
= & \mu F(\mu)-\int_{0}^{\mu} x f(x) d x-\mu[1-F(\mu)]+\int_{\mu}^{\infty} x f(x) d x \\
= & 2 \mu F(\mu)-2 \mu+2 \int_{\mu}^{\infty} x f(x) d x \\
= & 2 \mu F(\mu)-2 \int_{0}^{\mu} x f(x) d x
\end{aligned}
$$

and

$$
\begin{aligned}
& \delta_{2}(X)=\int_{0}^{M}(M-x) f(x) d x+\int_{M}^{\infty}(x-M) f(x) d x \\
= & M F(M)-\int_{0}^{M} x f(x) d x-M[1-F(M)]+\int_{M}^{\infty} x f(x) d x \\
= & -\mu+2 \int_{M}^{\infty} x f(x) d x \\
= & \mu-2 \int_{0}^{M} x f(x) d x
\end{aligned}
$$

Using p.d.f. (1.3), and the expression for the mean of QLD (1.3), we have

$$
\begin{aligned}
& \int_{0}^{\mu} x f(x) d x=\mu-\frac{\left\{\theta^{2} \mu^{2}+(\alpha+2) \theta \mu+(\alpha+2)\right\} e^{-\theta \mu}}{\theta(\alpha+1)} \\
& \int_{0}^{M} x f(x) d x=\mu-\frac{\left\{\theta^{2} M^{2}+(\alpha+2) \theta M+(\alpha+2)\right\} e^{-\theta M}}{\theta(\alpha+1)}
\end{aligned}
$$

Using expressions from (2.2.1), (2.2.2), (2.2.3) and (2.2.4) and little algebraic simplification, $\delta_{1}(\mathrm{X})$ and $\delta_{2}(\mathrm{X})$ of QLD (1.3), are obtained as

$$
\delta_{1}(X)=\frac{2(\theta \mu+\alpha+2) e^{-\theta \mu}}{\theta(\alpha+1)}
$$

and 


$$
\delta_{2}(X)=\frac{2\left\{\theta^{2} M^{2}+(\alpha+2) \theta M+(\alpha+2)\right\} e^{-\theta M}}{\theta(\alpha+1)}-\mu
$$

It can be easily verified that expressions (2.2.5) and (2.2.6) of QLD (1.3) reduce to the corresponding expressions of Lindley distribution at $\alpha=\theta$.

\subsection{Distribution of Order Statistics}

Let $\left(\mathrm{X}_{1}, \mathrm{X}_{2}, \ldots, \mathrm{X}_{\mathrm{n}}\right)$ be a random sample of size $\mathrm{n}$ from QLD (1.3). Let $X_{(1)}<X_{(2)}<\ldots<X_{(n)}$ denote the corresponding order statistics. The p.d.f. and the c.d.f. of the $\mathrm{k}$ th order statistic, say $\mathrm{Y}=\mathrm{X}_{(\mathrm{k})}$ are given by

$$
\begin{aligned}
& f_{Y}(y)=\frac{n !}{(k-1) !(n-k) !} F^{k-1}(y)\{1-F(y)\}^{n-k} f(y) \\
& f_{Y}(y)=\frac{n ! \theta(\alpha+\theta x) e^{-\theta x}}{(\alpha+1)(k-1) !(n-k) !} \sum_{l=0}^{n-k}\left(\begin{array}{c}
n-k \\
l
\end{array}\right)(-1)^{l} \times\left[1-\frac{1+\alpha+\theta x}{\alpha+1} e^{-\theta x}\right]^{k+l-1}
\end{aligned}
$$

and

$$
F_{Y}(y)=\sum_{j=k}^{n} \sum_{l=0}^{n-j}\left(\begin{array}{c}
n \\
j
\end{array}\right)\left(\begin{array}{c}
n-j \\
l
\end{array}\right)(-1)^{l}\left[1-\frac{1+\alpha+\theta x}{\alpha+1} e^{-\theta x}\right]^{j+l}
$$

It can be easily verified that the expressions for the p.d.f. and c.d.f. of the $\mathrm{k}$ th order statistics of QLD (1.3) reduce to the corresponding expressions for the p.d.f. and c.d.f. of the $\mathrm{k}$ th order statistics of Lindley distribution at $\alpha=\theta$.

$$
=\frac{n !}{(k-1) !(n-k) !} \sum_{l=0}^{n-k}\left(\begin{array}{c}
n-k \\
l
\end{array}\right)(-1)^{l} F^{k+l-1}(y) f(y)
$$

and

$$
\begin{aligned}
& F_{Y}(y)=\sum_{j=k}^{n}\left(\begin{array}{l}
n \\
j
\end{array}\right) F^{j}(y)\{1-F(y)\}^{n-j} \\
& =\sum_{j=k}^{n} \sum_{l=0}^{n-j}\left(\begin{array}{c}
n \\
j
\end{array}\right)\left(\begin{array}{c}
n-j \\
l
\end{array}\right)(-1)^{l} F^{j+l}(y),
\end{aligned}
$$

respectively, for $\mathrm{k}=1,2,3, \ldots, \mathrm{n}$.

Thus, the p.d.f. and the c.d.f of the $\mathrm{k}$ th order statistics of QLD (1.3) are obtained as

$$
B(p)=\frac{1}{p \mu} \int_{0}^{q} x f(x) d x=\frac{1}{p \mu}\left[\int_{0}^{\infty} x f(x) d x-\int_{q}^{\infty} x f(x) d x\right]=\frac{1}{p \mu}\left[\mu-\int_{q}^{\infty} x f(x) d x\right]
$$

and

$$
L(p)=\frac{1}{\mu} \int_{0}^{q} x f(x) d x=\frac{1}{\mu}\left[\int_{0}^{\infty} x f(x) d x-\int_{q}^{\infty} x f(x) d x\right]=\frac{1}{\mu}\left[\mu-\int_{q}^{\infty} x f(x) d x\right]
$$

respectively or equivalently

$$
B(p)=\frac{1}{p \mu} \int_{0}^{p} F^{-1}(x) d x
$$

and

$$
L(p)=\frac{1}{\mu} \int_{0}^{p} F^{-1}(x) d x
$$

respectively, where $\mu=E(X)$ and $q=F^{-1}(p)$.

The Bonferroni and Gini indices are thus defined as

$$
B=1-\int_{0}^{1} B(p) d p
$$

and

$$
G=1-2 \int_{0}^{1} L(p) d p
$$

respectively.

Using p.d.f. (1.3), we have

$$
\int_{q}^{\infty} x f(x) d x=\frac{\left\{\theta^{2} q^{2}+(\alpha+2) \theta q+(\alpha+2)\right\} e^{-\theta q}}{\theta(\alpha+1)}
$$

Now using equation (2.4.7) in (2.4.1) and (2.4.2), we have

$$
B(p)=\frac{1}{p}\left[1-\frac{\left\{\theta^{2} q^{2}+(\alpha+2) \theta q+(\alpha+2)\right\} e^{-\theta q}}{\alpha+2}\right]
$$




$$
L(p)=1-\frac{\left\{\theta^{2} q^{2}+(\alpha+2) \theta q+(\alpha+2)\right\} e^{-\theta q}}{\alpha+2}
$$

Now using equations (2.4.8) and (2.4.9) in (2.4.5) and (2.4.6), the Bonferroni and Gini indices of QLD (1.3) are obtained as

$$
\begin{aligned}
& B=1-\frac{\left\{\theta^{2} q^{2}+(\alpha+2) \theta q+(\alpha+2)\right\} e^{-\theta q}}{\alpha+2} \\
& G=-1+\frac{\left\{\theta^{2} q^{2}+(\alpha+2) \theta q+(\alpha+2)\right\} e^{-\theta q}}{\alpha+2}
\end{aligned}
$$

The Bonferroni and Gini indices of Lindley distribution are particular cases of the Bonferroni and Gini indices (2.4.10) and (2.4.11) of QLD (1.3) for $\alpha=\theta$.

\subsection{Renyi Entropy Measure}

The entropy of a random variable $\mathrm{X}$ is a measure of variation of uncertainty. A popular entropy measure is Renyi entropy (1961). If $\mathrm{X}$ is a continuous random variable having probability density function $f($.$) , then Renyi entropy is$ defined as

$$
T_{R}(\gamma)=\frac{1}{1-\gamma} \log \left\{\int f^{\gamma}(x) d x\right\}
$$

where $\gamma>0$ and $\gamma \neq 1$.

Thus, the Renyi entropy for QLD (1.3) can be obtained as

$$
\begin{aligned}
& T_{R}(\gamma)=\frac{1}{1-\gamma} \log \left[\int_{0}^{\infty}\left(\frac{\theta}{\alpha+1}\right)^{\gamma}(\alpha+\theta x)^{\gamma} e^{-\theta \gamma x} d x\right] \\
& =\frac{1}{1-\gamma} \log \left[\int_{0}^{\infty}\left(\frac{\theta}{\alpha+1}\right)^{\gamma} \alpha^{\gamma}\left(1+\frac{\theta}{\alpha} x\right)^{\gamma} e^{-\theta \gamma x} d x\right] \\
& =\frac{1}{1-\gamma} \log \left[\int_{0}^{\infty}\left(\frac{\alpha \theta}{\alpha+1}\right)^{\gamma} \sum_{j=0}^{\infty}\left(\begin{array}{l}
\gamma \\
j
\end{array}\right)\left(\frac{\theta}{\alpha} x\right)^{j} e^{-\theta \gamma x} d x\right] \\
& =\frac{1}{1-\gamma} \log \left[\left(\frac{\alpha \theta}{\alpha+1}\right)^{\gamma} \sum_{j=0}^{\infty}\left(\begin{array}{l}
\gamma \\
j
\end{array}\right)\left(\frac{\theta}{\alpha}\right)^{j} \int_{0}^{\infty} e^{-\theta \gamma x} x^{j+1-1} d x\right] \\
& =\frac{1}{1-\gamma} \log \left[\left(\frac{\alpha \theta}{\alpha+1}\right)^{\gamma} \sum_{j=0}^{\infty}\left(\begin{array}{l}
\gamma \\
j
\end{array}\right)\left(\frac{\theta}{\alpha}\right)^{j} \frac{\Gamma(j+1)}{(\theta \gamma)^{j+1}}\right] \\
& =\frac{1}{1-\gamma} \log \left[\sum_{j=0}^{\infty}\left(\begin{array}{l}
\gamma \\
j
\end{array}\right)\left(\frac{\alpha \theta}{\alpha+1}\right)^{\gamma}\left(\frac{\theta}{\alpha}\right)^{j} \frac{\Gamma(j+1)}{(\theta \gamma)^{j+1}}\right]
\end{aligned}
$$

The Renyi entropy of Lindley distribution is a particular case of the Renyi entropy of QLD (1.3) at $\alpha=\theta$.

\subsection{Stress-Strength Reliability}

The stress- strength reliability of a component describes the life of the component having random strength $\mathrm{X}$ subject to a random stress $\mathrm{Y}$. When the stress applied to the component exceeds the strength $(\mathrm{X}<\mathrm{Y})$, the component fails instantly and the component will function satisfactorily till $\mathrm{X}>\mathrm{Y}$. Therefore, $\mathrm{R}=\mathrm{P}(\mathrm{Y}<\mathrm{X})$ is a measure of the component reliability and is known as stress-strength parameter in reliability engineering. It has wide applications in almost all areas of knowledge especially in engineering such as structures, deterioration of rocket motors, static fatigue of ceramic components, aging of concrete pressure vessels etc.

Let $\mathrm{X}$ and $\mathrm{Y}$ be independent strength and stress random variables having QLD (1.3) with parameter $\left(\alpha_{1}, \theta_{1}\right)$ and $\left(\alpha_{2}, \theta_{2}\right)$ respectively. Then the stress-strength reliability $\mathrm{R}$ can be obtained as

$$
R=P(Y<X)=\int_{0}^{\infty} P(Y<X \mid X=x) f_{X}(x) d x
$$

$$
=\int_{0}^{\infty} f\left(x ; \alpha_{1}, \theta_{1}\right) F\left(x ; \alpha_{2}, \theta_{2}\right) d x
$$

$=1-\frac{\theta_{1}\left[2 \theta_{1} \theta_{2}+\left(\alpha_{1} \theta_{2}+\alpha_{2} \theta_{1}+\theta_{1}\right)\left(\theta_{1}+\theta_{2}\right)+\alpha_{1}\left(1+\alpha_{2}\right)\left(\theta_{1}+\theta_{2}\right)^{2}\right]}{\left(\alpha_{1}+1\right)\left(\alpha_{2}+1\right)\left(\theta_{1}+\theta_{2}\right)^{3}}$

The expression of stress-strength reliability of QLD reduces to the corresponding expression for stress-strength reliability of Lindley distribution at $\alpha_{1}=\theta_{1}$ and $\alpha_{2}=\theta_{2}$.

\section{Estimation of Parameters}

\subsection{Method of Moment Estimate (MOME) of Parameters}

Since the QLD (1.3) has two parameters to be estimated, the first two moments about origin are required to estimate its parameters. Using the first two moments about origin of QLD (1.3), we have

$$
\frac{\mu_{2}^{\prime}}{\left(\mu_{1}^{\prime}\right)^{2}}=K(\text { Say })=\frac{2(\alpha+3)(\alpha+1)}{(\alpha+2)^{2}}
$$

Equation (3.1.1) gives a quadratic equation in $\alpha$ as

$$
(2-k) \alpha^{2}+4(2-k) \alpha+2(3-2 k)=0
$$

Replacing $\mu_{1}^{\prime}$ and $\mu_{2}^{\prime}$ by their respective sample moments in (3.1.1), an estimate of $\mathrm{k}$ can be obtained and substituting the value of $k$ in equation (3.1.2), moment estimate $\tilde{\alpha}$ of $\alpha$ can be obtained. Substituting the moment estimate of $\alpha$ in the expression for the mean of QLD (1.3), moment estimate $\tilde{\theta}$ of $\theta$ can be obtained as

$$
\tilde{\theta}=\left(\frac{\alpha+2}{\alpha+1}\right) \frac{1}{\bar{x}}
$$




\subsection{Maximum Likelihood Estimate (MLE) of Parameters}

Let $\left(\mathrm{x}_{1}, \mathrm{x}_{2}, \mathrm{x}_{3}, \ldots, \mathrm{x}_{\mathrm{n}}\right)$ be a random sample of size $\mathrm{n}$ from QLD (1.3). Let $f_{x}$ be the observed frequency in the sample corresponding to $X=x(x=1,2,3, \ldots k)$ such that $\sum_{x=1}^{k} f_{x}=n$, where $\mathrm{k}$ is the largest observed value having non-zero frequency. The likelihood function, L of QLD (1.3) is given by

$$
L=\left(\frac{\theta}{\alpha+1}\right)^{2} \prod_{x=1}^{n}(\alpha+\theta x)^{f_{x}} e^{-n \theta \bar{x}}
$$

The natural $\log$ likelihood function is thus obtained as

$$
\ln L=n \ln \theta-n \ln (\alpha+1)+\sum_{x=1}^{k} f_{x} \ln (\alpha+\theta x)-n \theta \bar{x}
$$

where $\overline{\mathrm{x}}$ is the sample mean.

The two log likelihood equations are obtained as

$$
\begin{gathered}
\frac{\partial \ln L}{\partial \theta}=\frac{n}{\theta}+\sum_{x=1}^{k} \frac{x f_{x}}{\alpha+\theta x}-n \bar{x}=0 \\
\frac{\partial \ln L}{\partial \alpha}=-\frac{n}{\alpha+1}+\sum_{x=1}^{k} \frac{f_{x}}{\alpha+\theta x}=0
\end{gathered}
$$

The equations (3.2.3) and (3.2.4) do not seem to be solved directly. However, Fisher's scoring method can be applied to solve these equations iteratively. We have

$$
\begin{gathered}
\frac{\partial^{2} \ln L}{\partial \theta^{2}}=-\frac{n}{\theta^{2}}-\sum_{x=1}^{k} \frac{x^{2} f_{x}}{(\alpha+\theta x)^{2}} \\
\frac{\partial^{2} \ln L}{\partial \theta \partial \alpha}=-\sum_{x=1}^{k} \frac{x f_{x}}{(\alpha+\theta x)^{2}} \\
\frac{\partial^{2} \ln L}{\partial \alpha^{2}}=-\frac{n}{(\alpha+1)^{2}}-\sum_{x=1}^{k} \frac{f_{x}}{(\alpha+\theta x)^{2}}
\end{gathered}
$$

The maximum likelihood estimates $\hat{\theta}$ and $\hat{\alpha}$ of parameters $\theta$ and $\alpha$ are the solution of the following equations

$$
\left[\begin{array}{ll}
\frac{\partial^{2} \ln L}{\partial \theta^{2}} & \frac{\partial^{2} \ln L}{\partial \theta \partial \alpha} \\
\frac{\partial^{2} \ln L}{\partial \theta \partial \alpha} & \frac{\partial^{2} \ln L}{\partial \alpha^{2}}
\end{array}\right]_{\substack{\hat{\theta}=\theta_{0} \\
\hat{\alpha}=\alpha_{0}}}\left[\begin{array}{c}
\hat{\theta}-\theta_{0} \\
\hat{\alpha}-\alpha_{0}
\end{array}\right]=\left[\begin{array}{c}
\frac{\partial \ln L}{\partial \theta} \\
\frac{\partial \ln L}{\partial \alpha}
\end{array}\right]_{\substack{\hat{\theta}=\theta_{0} \\
\hat{\alpha}=\alpha_{0}}}
$$

where $\theta_{0}$ and $\alpha_{0}$ are initial values of $\theta$ and $\alpha$, preferably method of moment estimates of the parameters. These equations are solved iteratively till sufficiently close estimates of $\hat{\theta}$ and $\hat{\alpha}$ are obtained.

\section{Goodness of Fit to Real Lifetime Data- Sets}

The quasi Lindley distribution (QLD) has been fitted to a number of lifetime data- sets. In this section, we present the fitting of QLD to five real lifetime data-sets and compare its goodness of fit with exponential and Lindley distributions. The following five lifetime data-sets have been considered for comparing the goodness of fit of QLD with Lindley and exponential distributions.

Data set 1: This data set represents the lifetime's data relating to relief times (in minutes) of 20 patients receiving an analgesic and reported by Gross and Clark (1975, P. 105).

$1.1,1.4,1.3,1.7,1.9,1.8,1.6,2.2,1.7,2.7,4.1,1.8,1.5,1.2$, $1.43,1.7,2.3,1.62$

Data Set 2: This data set is the strength data of glass of the aircraft window reported by Fuller et al (1994):

$18.83,20.8,21.657,23.03,23.23,24.05,24.321,25.5$ $25.52,25.8,26.69,26.77,26.78,27.05,27.67,29.9,31.11$, $33.2,33.73,33.76,33.89,34.76,35.75,35.91,36.98,37.08$, 37.09, 39.58, 44.045, 45.29, 45.381

Data set 3: This data set represents the waiting times (in minutes) before service of 100 Bank customers and examined and analyzed by Ghitany et al (2008) for fitting the Lindley (1958) distribution.

$0.8,0.8,1.3,1.5,1.8,1.9,1.9,2.1,2.6,2.7,2.9,3.1,3.2,3.3$, 3.5, 3.6, 4.0, 4.1, 4.2, 4.2, 4.3, 4.3, 4.4, 4.4, 4.6, 4.7, 4.7, 4.8, $4.9,4.9,5.0,5.3,5.5,5.7,5.7,6.1,6.2,6.2,6.2,6.3$, $6.7,6.9,7.1,7.1,7.1,7.1,7.4,7.6,7.7,8.0,8.2,8.6,8.6,8.6$, $8.8,8.8,8.9,8.9,9.5,9.6,9.7,9.8,10.7,10.9,11.0,11.0,11.1$, $11.2,11.2,11.5,11.9,12.4,12.5,12.9,13.0,13.1,13.3,13.6$, $13.7,13.9,14.1,15.4,15.4,17.3,17.3,18.1,18.2,18.4,18.9$, $19.0,19.9,20.6,21.3,21.4,21.9,23.0,27.0,31.6,33.1,38.5$

Data Set 4: The data set represents the strength of $1.5 \mathrm{~cm}$ glass fibers measured at the National Physical Laboratory, England. Unfortunately, the units of measurements are not given in the paper, and they are taken from Smith and Naylor (1987)

$0.55,0.93,1.25,1.36,1.49,1.52,1.58,1.61,1.64,1.68$, $1.73,1.81,2.00,0.74,1.04,1.27,1.39,1.49,1.53,1.59,1.61$, $1.66,1.68,1.76,1.82,2.01,0.77,1.11,1.28,1.42,1.50,1.54$, $1.60,1.62,1.66,1.69,1.76,1.84,2.24,0.81,1.13,1.29$, $1.48,1.50,1.55,1.61,1.62,1.66,1.70,1.77,1.84,0.84,1.24$, $1.30,1.48,1.51,1.55,1.61,1.63,1.67$

1.701 .781 .89

Data Set 5: The data set is from Lawless (1982, p-228). The data given arose in tests on endurance of deep groove ball bearings. The data are the number of million revolutions before failure for each of the 23 ball bearings in the life tests and they are:

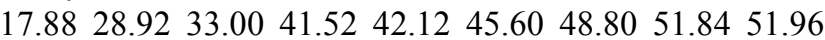
54.1255 .5667 .80

68.4468 .6468 .8884 .1293 .1298 .64105 .12105 .84127 .92 128.04173 .40 
In order to compare QLD, exponential and Lindley distributions, $-2 \ln \mathrm{L}$, AIC (Akaike Information Criterion), AICC (Akaike Information Criterion Corrected), BIC (Bayesian Information Criterion),K-S Statistics ( Kolmogorov-Smirnov Statistics) for five real lifetime data sets have been computed and presented in table 1. The formulae for computing AIC, AICC, BIC, and K-S Statistics are as follows:

$$
\begin{aligned}
& \mathrm{AIC}=-2 \ln L+2 k, \quad \mathrm{AICC}=\mathrm{AIC}+\frac{2 k(k+1)}{(n-k-1)}, \\
& \mathrm{BIC}=-2 \ln L+k \ln n \text { and } D=\operatorname{Sup}_{x}\left|F_{n}(x)-F_{0}(x)\right|,
\end{aligned}
$$

where $\mathrm{k}=$ the number of parameters, $\mathrm{n}=$ the sample size and $F_{n}(x)$ is the empirical distribution function. The best distribution is the distribution corresponding to the lower values of $-2 \ln \mathrm{L}, \mathrm{AIC}$, AICC, BIC, and K-S statistics.

\begin{tabular}{|c|c|c|c|c|c|c|c|c|}
\hline & \multirow{2}{*}{ Model } & \multicolumn{2}{|c|}{ Estimate of Parameters } & \multirow{2}{*}{$-2 \ln L$} & \multirow{2}{*}{ AIC } & \multirow{2}{*}{ AICC } & \multirow{2}{*}{ BIC } & \multirow{2}{*}{$\begin{array}{l}\text { K-S } \\
\text { Statistics }\end{array}$} \\
\hline & & $\hat{\theta}$ & $\hat{\alpha}$ & & & & & \\
\hline \multirow[t]{3}{*}{ Data 1} & Lindley & 0.816118 & & 60.50 & 62.50 & 62.72 & 63.49 & 0.34 \\
\hline & Exponential & 0.526316 & & 65.67 & 67.67 & 67.90 & 68.67 & 0.39 \\
\hline & QLD & 1.545110 & -0.483393 & 40.71 & 44.71 & 45.41 & 46.70 & 0.20 \\
\hline \multirow[t]{3}{*}{ Data 2} & Lindley & 0.062988 & & 253.99 & 255.99 & 256.13 & 257.42 & 0.33 \\
\hline & Exponential & 0.032455 & & 274.53 & 276.53 & 276.67 & 277.96 & 0.43 \\
\hline & QLD & 0.103985 & -0.546267 & 231.82 & 235.82 & 236.25 & 238.69 & 0.30 \\
\hline \multirow[t]{3}{*}{ Data 3} & Lindley & 0.186571 & & 638.07 & 640.07 & 640.12 & 642.68 & 0.06 \\
\hline & Exponential & 0.101245 & & 658.04 & 660.04 & 660.08 & 662.65 & 0.16 \\
\hline & QLD & 0.196209 & 0.066138 & 635.75 & 639.75 & 639.87 & 639.75 & 0.05 \\
\hline \multirow[t]{3}{*}{ Data 4} & Lindley & 0.996116 & & 162.56 & 164.56 & 164.62 & 166.70 & 0.37 \\
\hline & Exponential & 0.663647 & & 177.66 & 179.66 & 179.73 & 181.80 & 0.40 \\
\hline & QLD & 2.146473 & -0.552445 & 91.56 & 95.56 & 95.63 & 97.36 & 0.36 \\
\hline \multirow{2}{*}{ Data 5} & Exponential & 0.013845 & & 242.87 & 244.87 & 245.06 & 246.01 & 0.26 \\
\hline & QLD & 0.035434 & -0.358716 & 223.52 & 227.52 & 228.12 & 229.79 & 0.10 \\
\hline
\end{tabular}

Table 1. MLE's, - 2ln L, AIC, AICC, BIC, K-S Statistics of the fitted distributions of data sets 1-5.

It is obvious from the fitting of QLD, Lindley and exponential distributions in the table 1 that QLD gives much closer fit than Lindley and exponential distributions in all data- sets, and therefore QLD can be preferred over Lindley and exponential distributions for modeling lifetime data-sets from biomedical science, engineering and other fields of knowledge.

\section{Concluding Remarks}

In the this paper some important mathematical and statistical properties including moment generating function, mean deviations about mean and median, order statistics, Bonferroni and Lorenz curves, Renyi entropy measure and stress strength reliability of quasi Lindley distribution (QLD) of Shanker and Mishra (2013 a) have been derived and discussed. The distribution has been fitted to some real lifetime data-sets to test its goodness of fit over exponential and Lindley distributions. It is clear from the fitting of QLD that it gives better fitting than exponential and Lindley distributions and hence QLD can be recommended over exponential and Lindley distributions for modeling real lifetime data-sets from biomedical science and engineering.

\section{References}

[1] Alkarni, S. (2015): Extended Power Lindley distribution-A new Statistical model for non-monotone survival data, European journal of statistics and probability, 3(3), 19-34.
[2] Ashour, S. and Eltehiwy, M. (2014): Exponentiated Power Lindley distribution, Journal of Advanced Research.

[3] Bakouch, H.S., Al-Zaharani, B. Al-Shomrani, A., Marchi, V. and Louzad, F. (2012): An extended Lindley distribution, Journal of the Korean Statistical Society, 41, 75-85.

[4] Bonferroni, C.E. (1930): Elementi di Statistca generale, Seeber, Firenze.

[5] Deniz, E. and Ojeda, E. (2011): The discrete Lindley distribution-Properties and Applications, Journal of Statistical Computation and Simulation, 81, 1405-1416.

[6] Elbatal, I., Merovi, F. and Elgarhy, M. (2013): A new generalized Lindley distribution, Mathematical theory and Modeling, 3 (13), 30-47.

[7] Fuller, E.J., Frieman, S., Quinn, J., Quinn, G., and Carter, W. (1994): Fracture mechanics approach to the design of glass aircraft windows: A case study, SPIE Proc 2286, 419-430.

[8] Ghitany, M.E., Atieh, B. and Nadarajah, S. (2008): Lidley distribution and its Application, Mathematics Computing and Simulation, 78, 493-506.

[9] Ghitany, M., Al-Mutairi, D., Balakrishnan, N. and Al-Enezi, I. (2013): Power Lindley distribution and associated inference, Computational Statistics and Data Analysis, 64, 20-33.

[10] Gross, A. J. and Clark, V. A. (1975): Survival Distributions: Reliability Applications in the Biometrical Sciences, John Wiley, New York.

[11] Lawless, J.F. (1982): Statistical models and methods for lifetime data, John Wiley and Sons, New York. 
[12] Lindley, D.V. (1958): Fiducial distributions and Bayes' theorem, Journal of the Royal Statistical Society, Series B, 20, 102-107.

[13] Liyanage, G.W. and Pararai. M. (2014): A generalized Power Lindley distribution with applications, Asian journal of Mathematics and Applications, 1-23.

[14] Merovci, F. (2013): Transmuted Lindley distribution, International Journal of Open Problems in Computer Science and Mathematics, 6, 63-72.

[15] Nadarajah, S., Bakouch, H.S. and Tahmasbi, R. (2011): A generalized Lindley distribution, Sankhya Series B, 73, 331359.

[16] Oluyede, B.O. and Yang, T. (2014): A new class of generalized Lindley distribution with applications, Journal of Statistical Computation and Simulation, 85 (10), 2072-2100.

[17] Pararai, M., Liyanage, G.W. and Oluyede, B.O. (2015): A new class of generalized Power Lindley distribution with applications to lifetime data, Theoretical Mathematics \&Applications, 5(1), 53-96.

[18] Renyi, A. (1961): On measures of entropy and information, in proceedings of the $4^{\text {th }}$ berkeley symposium on Mathematical Statistics and Probability, 1, 547-561, Berkeley, University of California press.

[19] Shanker, R. and Mishra, A. (2013 a): A quasi Lindley distribution, African Journal of Mathematics and Computer Science Research, 6(4), 64-71.

[20] Shanker, R. and Mishra, A. (2013 b): A two-parameter Lindley distribution, Statistics in Transition-new series, 14 (1), 45-56.
[21] Shanker, R. and Amanuel, A.G. (2013): A new quasi Lindley distribution, International Journal of Statistics and Systems, 8 (2), 143-156.

[22] Shanker, R., Sharma, S. and Shanker, R. (2013): A twoparameter Lindley distribution for modeling waiting and survival times data, Applied Mathematics, 4, 363-368.

[23] Shanker, R., Hagos, F, and Sujatha, S. (2015 a): On modeling of Lifetimes data using exponential and Lindley distributions, Biometrics \& Biostatistics International Journal, 2 (5), 1-9.

[24] Shanker, R., Hagos, F. and Sharma, S. (2015 b): On Twoparameter Lindley distribution and its Applications to Model Lifetime data, Biometrics and Biostatistics International Journal, 3(2), 1-8.

[25] Sharma, V., Singh, S., Singh, U. and Agiwal, V. (2015): The inverse Lindley distribution- A stress-strength reliability model with applications to head and neck cancer data, Journal of Industrial \&Production Engineering, 32 (3), 162-173.

[26] Singh, S.K., Singh, U. and Sharma, V.K. (2014): The Truncated Lindley distribution- inference and Application, Journal of Statistics Applications\& Probability, 3(2), 219-228.

[27] Smith, R.L, and Naylor, J.C. (1987): A comparison of Maximum likelihood and Bayesian estimators for the three parameter Weibull distribution, Applied Statistics, 36, 358-369.

[28] Zakerzadeh, H. and Dolati, A. (2009): Generalized Lindley distribution, Journal of Mathematical extension, 3 (2), 13-25 